Editorial

\title{
The implications and applications of learning via instructions
}

\begin{abstract}
A B S T R A C T
Whereas psychology knows a long tradition of studies that focused on the role of practice and training in acquiring new skills or knowledge, systematic studies into learning via instructions remain relatively scarce. This is surprising given the tremendous influence instructions have on human behavior and cognition. In recent years, however, a (re)new(ed) interest into learning via instructions resulted in new paradigms and findings that can inspire future research in this understudied domain. We offer a brief overview of the articles in this special issue, which present some of the latest empirical developments dedicated to unraveling the implications and applications of learning via instructions. The special issue offers insights into the dynamics underlying the assimilation of new instructions and highlights the strengths and limitations of what can be achieved on the basis of instructions. Furthermore, the different studies showcase various examples of recent methodological advances in testing the effects of instructions. Finally, this special issue shows how different fields in psychology share similar questions on the role of instructions in human behavior, suggesting that this topic should no longer be considered as a subsidiary of these different fields, but as a research field on its own.
\end{abstract}

The ability to share and use instructions is thought to separate humans from other animal species. Using language, we can quickly share knowledge that allows us to bypass trial-and-error learning. This way, instructions are a prime catalyst of human behavior. Instructions determine our functioning in a whole spectrum of situations, ranging from the execution of a single movement to complex interpersonal interactions, which define a society. Yet, despite its unequivocal importance, the dynamics underlying the interplay between instructions and behavior remain a blind spot in our understanding of human cognition. Whereas research in psychology knows a long tradition of studies that focused on the role of practice or training in acquiring new skills or knowledge, systematic studies into learning via instructions remain relatively scarce.

In recent years, a (re)new(ed) interest into learning via instructions resulted in new paradigms and findings. Interestingly, these endeavors originated, more or less in parallel, in different domains of psychology, seemingly addressing different questions on the role of instructions in human behavior. The diversity of research on instructions was recently illustrated by the 27th Attention \& Performance meeting on "The Power of Instructions", bringing together lead scientists from different domains in psychology and cognitive neurosciences. This meeting resulted in a special section in Neuroscience \& Biobehavioral Reviews, edited by Marcel Brass and Jan De Houwer, featuring six theoretical reviews and a discussion paper on instructions (Brass, Liefooghe, Braem, \& De Houwer, in press; Cole, Braver, and Meiran, in press; De Houwer, Hughes, \& Brass, in press; Hommel and Colzato, in press; Koban, Jepma, Geuter, \& Wager, in press; Landry, Lifshitz, \& Raz, in press; Terhune, Cleeremans, Raz, \& Lynn, in press). In parallel, we organized this special issue which aimed at presenting the latest empirical developments that are dedicated to unraveling the implications and applications of learning via instructions. The thirteen articles in this issue originate from both an initial call for contributions to all attendees of the 27th Attention \& Performance meeting, as well as an open call. The present editorial offers a brief overview of the thirteen contributions in this special issue. Taking abstraction from the different goals they pursue, we wish to highlight that they convey similar conclusions on the strength and limits of learning via instructions, and sometimes endorse similar methodological approaches.

An attractive feature of instructions is that they offer a fast route to learning compared to trial-and-error learning. Whereas such a claim seems parsimonious, differences between both types of learning have not been systematically investigated. Ruge, Karcz, Mark, Martin, Zwosta, and Wolfensteller (in this issue) offer a first start in this direction by comparing the gain in efficiency associated with learning via instructions relative to trial-and-error learning. Not only do their findings emphasize the power of instructions in attaining skilled behavior, but they also demonstrate that trial-and-error learning negatively impacts the accumulation of skill through the formation of erroneous memory traces. Learning via instructions is, however, possibly more resource demanding than learning via overt practice. This is illustrated by Cole, Patrick, Meiran, \& Braver (in this issue) who argue that the rapid learning of novel tasks from instructions depends upon the formation of new associations between long-term memory representations, which must then be actively maintained to enable successful task implementation. Consequently, they hypothesize that the formation of new tasks on the basis of instructions relies more heavily on a proactive mode of cognitive control compared to the retrieval of already practiced 
tasks. In line with their predictions, Cole and colleagues document the presence of a "novelty cost", which is associated with the assimilation of new instructions and indicative of a key role for proactive cognitive control in the ability to rapidly learn novel tasks from instructions.

Although learning via instructions is costly, its power to induce new representations and bias cognition is impressive. This can be illustrated by several demonstrations across different domains of psychology. For instance, within the domain of fear conditioning, Deltomme, Mertens, Tibboel, and Braem (in this issue) investigated whether stimuli merely instructed to be fear-relevant can bias visual attention, even when the fear relation was never experienced before. Instructing participants that a stimulus could be followed by an electrical stimulation at some later stage of the experiment (i.e., instructed fear), was sufficient to bias visual attention allocated to that stimulus in a dot-probe paradigm. These findings demonstrate that instructed fear can also elicit an automatic bias in visual attention, an observation commonly used to show automaticity after experience-based learning. Another example illustrating the power of instructions comes from a cognitive control study by Desender (in this issue). Specifically, using a response-conflict paradigm, Desender investigated whether a proportion congruency effect could be induced on the basis of instructions. In conflict paradigms, it is often observed that the difference between more difficult incongruent trials (where task-relevant and -irrelevant information activate conflicting responses) and easier congruent trials is reduced when the proportion of incongruent trials is high, relative to when most of the trials are congruent. Desender tested whether instructions can be used to induce a proportion congruency effect, while holding the actual proportion of congruent trials constant. His findings indicate that different types of instructions can modulate the size of the congruency effect. Interestingly, a diffusion-model analysis suggests that these different instructions are able to induce a proportion congruency effect through different mechanisms. Related, but now in the field of social cognition, Pilditch and Custers (in this issue) investigated how communicated beliefs (or instructions) interact with the integration of first-hand evidence. Previous research revealed that people are more inclined to seek out confirming evidence when they are motivated to uphold a communicated belief. In their study, Pilditch and Custers explore whether merely communicated beliefs affect evidence integration over time when it is not of interest to uphold the belief and all evidence is readily available. Their results show that while communicated beliefs predict initial choices, they only bias subsequent choices when supported by initial evidence. Merely communicated beliefs can thus prevail even when not supported by long run evidence, and in the absence of a motivation to uphold them.

The powerful impact of instructions is also demonstrated in paradigms using implementation intention manipulations. In this literature, implementation intentions are typically contrasted with more generic goal intentions in order to investigate the added value of the formation of an ifthen plan on the basis of instructions. For instance, Bieleke, Legrand, Mignon, and Gollwitzer (in this issue) tested the extent to which an implementation intention - contrasted with a goal intention - generalizes beyond the conditional event specified by this implementation intention. Their results indicate that implementation intentions can indeed generalize to non-instructed but similar events and even impair performance when these similar events require a different response. It is clear that such generalization again exemplifies the strength by which instructions can modulate behavior. In a similar vein, Koban, Kusko, and Wager (in this issue) studied how explicit fear learning contributes to fear generalization and concluded that conscious expectations and higher-order inference play an important role. Although their study did not manipulate instructions directly, they discuss how their work could offer the basis for subsequent instruction studies by hypothesizing that instructed fear should profoundly impact fear generalization.

Whereas the power of instructions has been demonstrated across different studies, this power seems not without limits and some effects seem to remain a function of experience-based learning. Meier and Rey-Mermet (in this issue) illustrate this point by investigating online slowing in prospective memory tasks. In a prospective memory task, instructions are used to define an appropriate target event as a retrieval cue. Such ongoing task slowing has been mainly considered as a measure of strategic monitoring for the prospective memory cues, which is induced via instructions. Nevertheless, Meier and Rey-Mermet carefully compared different instruction conditions and demonstrate that task slowing is an after-effect of actually responding to prospective memory cues, rather than a strategic slowing induced by the instructions. The critical role of overt experience can also be illustrated by the work of Pfeuffer, Moutsopoulou, Waszak, and Kiesel (in this issue). These authors started from the notion that the repeated co-occurrence of stimuli and responses during overt practice leads to stronger stimulus-response associations. Accordingly, they investigated whether the repeated instruction of such co-occurrence also leads to stronger associations. Interestingly, whereas such strengthening was observed for overt practice, it was absent for learning via instructions. These findings thus indicate that overt practice and learning via instructions are underlain by different mechanisms. In a similar vein, Theeuwes, Liefooghe, De Schryver, and De Houwer (in this issue) compared the application of new instructions following overt practice, covert practice (i.e., motor imagery), or no practice. Their findings demonstrate that motor imagery can improve the application of newly instructed stimulus-response mappings, but not to the same degree as overt practice. They conclude that overt practice, motor imagery, and learning via instructions lead to qualitatively different representations. A final example indicating that instructions are not almighty is provided by Verhoeven, Kindt, Zomer, and de Wit (in this issue), who suggest that the role of instructions in overcoming habitual behavior is limited. These authors investigated the role of instructed goal intentions and implementation intentions in overriding habitual responses in the slips-of-action tasks. In this task, participants are required to selectively respond to outcomes that are still valuable, while suppressing responding to outcomes that are no longer valuable (i.e., going against learned stimulus-response associations). Implementation intentions were effective in helping to suppress previously learned associations, but only had a beneficial effect compared to goal intentions when the outcomes of these associations were abstract and had no intrinsic value to the participants.

Last, some studies more explicitly investigated the impact of different instruction types on specific forms of experience-based learning, such as approach/avoidance training or stimulus-response versus category-response learning (see below). For example, within the field of implicit evaluations, Van Dessel, De Houwer, and Smith (in this issue) investigated whether the effect of approach-avoidance instructions on implicit evaluation depends on the type of relational information embedded in these instructions. Participants either received instructions that they would move towards a certain non-word and move away from another non-word (self-agent instructions), or instructions that one non-word would move towards them and the other non-word would move away from them (stimulus-agent instructions). Self-agent instructions produced stronger effects than stimulusagent instructions on implicit evaluations of the non-words. These findings suggest that propositional processes play an important role in effects of approach-avoidance instructions on implicit evaluation and in implicit evaluation in general. Using a related approach, Longman, Milton, Will, and Verbruggen (in this issue) demonstrate how different instructions differently biased category learning. When category-to-response mappings were instructed, training mainly resulted in the formation of category-response associations and to a far less extent in the formation category-stimulus or stimulus-response associations. However, when stimulus-response mappings were instructed without mentioning category structures, the evidence for stimulus-response learning outweighed the evidence for category-response learning, again indicating the importance of instructions in learning.

To conclude, the present special issue offers new insights into the dynamics underlying the assimilation of new instructions. It highlights both the strengths and limitations of what can be achieved on the basis of instructions and demonstrates various examples of recent methodological advances in research on instructions. It would be presumptuous to argue that the various contributions in this special issue fill the conceptual gap in our 
understanding of how instructions impact behavior. Nevertheless, they illustrate the growing interest in this topic and help building a much-needed stronger empirical foundation, which will ultimately allow for better theorizing on learning via instructions. Finally, this special issue shows that different fields in psychology share similar questions on the role of instructions in human behavior, suggesting that this topic should no longer be considered as a subsidiary of these different fields, but as a research field on its own.

\section{References}

Bieleke, M., Legrand, E., Mignon, A., \& Gollwitzer, P. M. (2017). More than planned: Implementation intention effects in non-planned situations. (this issue).

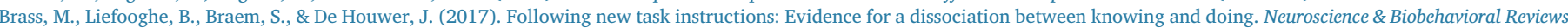
(in press).

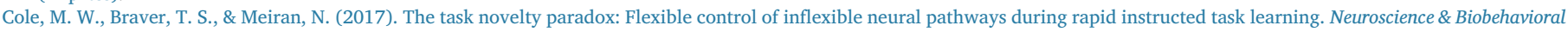
Reviews (in press).

Cole, M. W., Patrick, L. M., Meiran, N., \& Braver, T. S. (2017). A role for proactive control in rapid instructed task learning. (this issue).

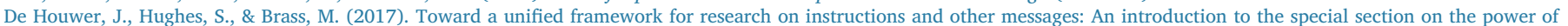
instructions. Neuroscience and Biobehavioral Reviews (in press).

Deltomme, B., Mertens, G., Tibboel, H., \& Braem, S. (2017). Instructed fear stimuli bias visual attention. (this issue).

Desender, K. (2017). Different mechanisms can account for the instruction induced proportion congruency effect. (this issue).

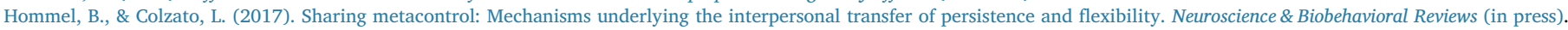

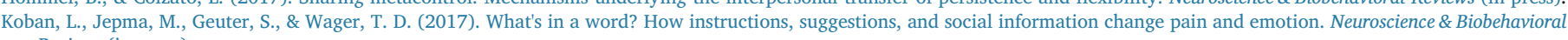
Reviews (in press).

Koban, L., Kusko, D., \& Wager, T. D. (2017). Generalization of learned pain modulation depends on explicit learning. (this issue).

Landry, M., Lifshitz, M., \& Raz, A. (2017). Brain correlates of hypnosis: A systematic review and meta-analytic exploration. Neuroscience \& Biobehavioral Reviews (in press).

Longman, C. S., Milton, F., Will, A. J., \& Verbruggen, F. (2017). Transfer of learned category-response associations is modulated by instruction. (this issue).

Meier, B., \& Rey-Mermet, A. (2017). After-effects without monitoring costs: The impact of prospective memory instructions on task switching performance. (this issue).

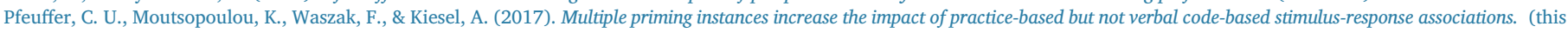
issue).

Pilditch, T. D., \& Custers, R. (2017). Communicated beliefs about action-outcomes: The role of initial confirmation in the adoption and maintenance of unsupported beliefs. (this issue). Ruge, H., Karcz, T., Mark, T., Martin, V., Zwosta, K., \& Wolfensteller, U. (2017). On the efficiency of instruction-based rule encoding. (this issue).

Terhune, D., Cleeremans, A., Raz, A., \& Lynn, S. J. (2017). Hypnosis and top-down regulation of consciousness. Neuroscience \& Biobehavioral Reviews (in press).

Theeuwes, M., Liefooghe, B., De Schryver, M., \& De Houwer, J. (2017). The role of motor imagery in learning via instructions. (this issue).

Van Dessel, P., De Houwer, J., \& Smith, C. T. (2017). Relational information moderates approach-avoidance instruction effects on implicit evaluation. (this issue).

Verhoeven, A. A. C., Kindt, M., Zomer, C. L., \& de Wit, S. (2017). An experimental investigation of breaking learnt habits with verbal implementation intentions. (this issue).

Baptist Liefooghe ${ }^{*, 1}$

Ghent University, Ghent, Belgium

E-mail address: baptist.liefooghe@ugent.be

Senne Braem ${ }^{2}$

Ghent University, Ghent, Belgium

Nachshon Meiran ${ }^{3}$

Ben-Gurion University of the Negev, Beer-Sheva, Israel

\footnotetext{
* Corresponding author at: H. Dunantlaan 2, B-9000 Ghent, Belgium.

${ }^{1}$ BL was supported by grant BOF16/MET_V/002 of Ghent University.

${ }^{2}$ SB was supported by grant $12 \mathrm{~K} 6316 \mathrm{~N}$ of the FWO - Research Foundation Flanders.

${ }^{3} \mathrm{NM}$ was supported was supported by grant 2015186 from the USA-Israel Bi-National Science Foundation given in collaboration to Todd S. Braver and Michael W. Cole.
} 